\title{
FIBER OPTICAL ACOUSTIC MONITORING SYSTEM OVER LARGE COASTAL SUB-WATER AREAS BASED ON LOW COHERENCE LASERS
}

\author{
Dimitar Stoyanov, Angel Popov*, \\ Tinko Eftimov and Stefan Vodenicharov \\ Institute of Metal Science Equipment and Technologies \\ with Hydro- and Aerodynamics Centre "Acad. Angel Balevski", \\ Bulgarian Academy of Sciences \\ 67, Shipchenski Prohod Blvd, 1574 Sofia, Bulgaria, \\ e-mails:dvstoyan@ie.bas.bg; angelsashev@gmail.com; teftimov@abv.bg
}

\begin{abstract}
The present study reports on an acoustic fiber optic sensor system with applications in the development of up to date stationary systems for complex acoustic monitoring of remote marine and land areas having no power supply of the acoustic sensors with capabilities for remote intruder detection and no detectability of the system by non-authorized external observers, immunity to electromagnetic noise and intentional jamming etc.

Keywords: acoustic fiber optic system, complex acoustic monitoring of remote marine and land areas, piezoelectric sensors.
\end{abstract}

\section{INTRODUCTION}

This work presents the results from the development and experimental studies of an acoustic fiber optic system which can find applications in the construction of modern stationary systems for complex acoustic monitoring of remote marine and land areas without power supply for the acoustic sensors and with a capability for remote detection of unknown intruders and no detectability of the system presence by non-authorized observers, insensitivity to intentional electromagnetic jamming etc.

Recent years witnessed progress in the use of the unique acoustic sensing capabilities offered by optical fibers for which sensitivity reached that of most widely used piezoelectric acoustic sensors and demonstrated advantages at ultralow frequencies. The need for an electric power supply for amplifiers of

\footnotetext{
${ }^{*}$ Corresponding author.

DOI: 10.7546/EngSci.LVIII.21.01.01
}

Engineering Sciences, LVIII, 2021, No. 1 
the conventional piezoelectric sensors leads to essential complications to the development of modern acoustic systems for complex monitoring over large remote marine and land areas.

A fiber optic device containing a broadband depolarized source, an input Michelson interferometer, a Y-splitter and a Fabry-Perot sensor resonator for the detection of seismic waves has been known for twenty years [1]. In this device the light from the broadband light source which is further depolarized passes through the input Michelson interferometer and the first port of the Y-splitter to reach the sensing Fabry-Perot interferometer, while the reflected signal is received via the second port of the Y-splitter and is further analyzed. A disadvantage of the device is the use of a broadband source, the relatively short sensor length which limits sensitivity and the possibility for a coherent detection especially for interrogating of remote acoustic sensors. Reference [2] describes a device using a coherent source, a fiber optic isolator, a splitter, an input fiber to a Fabry-Perot resonator encapsulated in a box with a highly sensitive to external longitudinal vibrations membrane and a photodetector unit in the reverse direction. Light from the single frequency laser traverses an optical isolator and through the first port of an X-splitter reaches a FabryPerot sensing resonator the reflected light from which is detected at the second port of the splitter. The disadvantage in this case is the lower sensitivity to weak acoustic signals due to the presence of mechanical components such as the membrane, the acoustic waveguide etc. Another important disadvantage is the incapability of creating networks of serially arranged sensors under mechanical stress which reduces the reliability of the system. Another known device [3, 4] has such disadvantages as:

a) limited sensitivity due to the fact that detection is on the slope of the transmission spectrum of the Fabry-Perot interferometer;

b) limited length of the lead-in fiber;

c) difficulties to multiplex multisensory systems.

Another known device [5] consists of serially connected time multiplexed Mach-Zehnder interferometers using 1\% fiber optic X-splitters. Light from the laser source is directed through the $\mathrm{X}$-coupler to a series of time multiplexed Mach-Zehnder interferometers, next to a modulated Mach-Zehnder interferometer and then to the detector system. The disadvantages are the complicated and costly construction, the use of many couplers which impose essential limitations to the development of monitoring systems over larger territories. 


\section{DESCRIPTION AND OPERATION OF THE ACOUSTIC SYSTEM}

An essential advantage of the proposed acoustic fiber optic system is the possibility to be deployed over distances as large as 10 to $20 \mathrm{~km}$ and above from the transmitter/receiver unit which together with interrogation unit make the system for acoustic monitoring marine and land areas. The link of the acoustic sensors with the transceiver/receiver unit is via a low-loss $(\leq 0.2 \mathrm{~dB} / \mathrm{km})$ optical fiber thus making the sensor system all-optical eliminating the need for electrical powering of the remote underwater amplifiers. Thus, it is the development of low-loss passive sensing networks for long-term complex acoustic monitoring of remote marine and land areas. The optical sensor systems for acoustic monitoring with a sensitivity over a section $\Delta l$ as a rule are based on coherent detection methods using sources of coherence length $L_{c}$ that fulfill the condition:

$$
K_{c o h}=\left(L_{c} / \Delta l\right)>1 .
$$

Typically, the reference signal is formed in the basic unit of the coherent system in which the length of the reference channel is $l_{\text {ref }} \ll L_{c}$. The present work refers to systems at a distance $L_{\text {sys }}$ from the basic unit for which the length of the signal channel is $l_{\text {sig }} \sim 2 L_{\text {sys }} n_{o} \gg l_{\text {ref }}$ where $n_{0}$ is the fiber core refractive index. For a higher efficiency of the coherent detection of the whole system the condition $K_{c o h}=\left(L_{c} / l_{\text {sig }}\right)>1$ has to be fulfilled from where the minimum coherence length can be estimated as $L_{c, \min }>2 L_{s y s} n_{0}$. For the acoustic sensor at a distance of $L_{\text {sys }}=20 \mathrm{~km}$ and $n_{0}=1.5$ the laser coherence length will have to be $L_{c}>60 \mathrm{~km}$. This leads to unacceptably high requirements to laser parameters and prices, an increase in the complexity of the acoustic monitoring systems and their maintenance.

It is the objective of the present work to develop a new fiber optic acoustic sensor system in which to minimize the difference between the signal and reference optical paths $l_{\text {sig }}$ and $l_{\text {ref }}$ of the signal and reference channels correspondingly:

$$
\Delta l=l_{\text {sig }}-l_{\text {ref }} \rightarrow 0,
$$

and to ensure conditions that the optical paths in both channels coincide in all part of the system outside the very acoustical system, i.e., to fulfill the condition

$$
l_{\text {sig }} \approx l_{\text {ref }}+l_{\text {sens }},
$$

where $l_{\text {sens }}$ is the optical path in the acoustic sensor. Then the condition

Engineering Sciences, LVIII, 2021, No. 1 
$\Delta l \sim l_{\text {sens }}$ can be fulfilled and for $K_{c o h}$ we obtain:

$$
K_{\text {coh }}=\left(L_{c} / l_{\text {sens }}\right)>1 \text {. }
$$

Assuming an acceptable value of the optical path in the sensor $l_{\text {sens }} \leq 20 \mathrm{~m}$, for the minimum optical coherence length of the laser we obtain the value $l>20 \mathrm{~m}$, from which the acceptable frequency stability is in the limits $\Delta f_{\text {las }} \leq(3-5) \mathrm{MHz}$ for which the condition (3) can be fulfilled. These values are typical for the routinely used and affordable single frequency telecommunication laser diodes. An important consequence from the fulfillment of the condition (3) is through the proper choice of laser diodes the possibility to build unique multiplexed sensor networks of a large number of remote independent acoustic sensors and simultaneously preserve a high level of efficiency of coherency detection.

The subject of our study is the practical realization of new efficient systems for acoustic monitoring in the case of large distance to the acoustic sensor based on the above-described conditions. On this basis a variety of technical realizations of acoustical optical fiber devices are possible which will widen the areas of application of the systems for remote acoustic monitoring such as border and port security, maritime and geophysical monitoring etc. An important advantage is that the high optical sensitivity and efficiency of the acoustic optical fiber sensors are realized with an all-optical system avoiding electrical power supply to the acoustical sensors which is caused by the possibility to have greater distances between the basic unit of the transmitter/receiver and the acoustic sensor transducer. Several other advantages of the proposed system can be outlined such as simplicity of the optical scheme, use of lower coherence and low-cost laser diodes, possibility to develop multisensory systems and networks described below.

Figure 1 represents the basic scheme of the fiber optic sensor with a single sensor which can be from 10 to above $20 \mathrm{~km}$ away from the basic interrogation unit. This simple arrangement can become a building block for time and spectrally multiplexed sensor systems that are appropriate to monitor large maritime areas of more than $1000 \mathrm{~km}^{2}$.

We next present a more detailed description of the system. In Figure 1, the system consists of a transmitter/receiver unit 1 with a lower coherency single frequency laser 2 , a fiber optic circulator 3 with input $3 \mathrm{a}$, input-output $3 \mathrm{~b}$ and output $3 \mathrm{c}$, as well as an optical detector 4 with an output to the interrogation unit of the acoustic monitoring. Through the input-output $3 \mathrm{~b}$ of the optical circulator 3 , the transmitter/receiver 1 is connected optically through a leadin/lead-out fiber of definite length 5 to the acoustic sensor 6 , consisting of 




Fig. 1. Basic scheme of a single sensor fiber optic system

a serially connected semi-transparent mirror 7 with a specified transmission coefficient, a fiber optic transducer 8 , a polarization controller 9 and a reflector 10 with a specified reflection coefficient.

The system functions in the following way. The single frequency laser 2 emits a continuous optical path at a single frequency $\omega_{\text {las }}=2 \pi f_{\text {las }}$, whose electric field $E_{\text {las }}(t)$ can be represented in the form:

$$
E_{\text {las }}(t)=E_{0} \exp \left[-j\left(\omega_{\text {las }} t+\varphi_{\text {las }}(t)\right)\right],
$$

where $E_{0}$ is the amplitude, while $\varphi_{\text {las }}(t)$ are the slow fluctuations of the optical phase. It is assumed that the frequency $\omega_{\text {las }}$ of the laser diode fluctuates within the limits $\omega_{\text {las }} \pm \Delta \omega_{\text {las }} / 2$ while the coherence length $L_{c}$ is chosen according to (3). From the input $3 \mathrm{a}$, and the output-input $3 \mathrm{~b}$ of the circulator 3 the continuous light from the laser 3 is passed through the optical fiber 5 to the acoustical sensor 6 . Upon reaching the partially transparent mirror 7 of a fixed transmission, part of the light is reflected in the reverse direction and after reaching the output-input $3 \mathrm{~b}$ of the circulator 3 through the output $3 \mathrm{c}$ is passed to the photosensitive area of the coherent detector 4 as a reference signal, the amplitude of which is given with the expression:

$$
E_{\text {ref }}(t)=\left(1-k_{\text {mir }}\right) k_{\text {ref }} E_{0} \exp \left[-j\left(\omega_{\text {las }} t+\varphi_{\text {las }}(t)+\Phi_{\text {ref }}\left(l_{\text {ref }}\right)\right)\right],
$$

where $k_{m i r}$ is the transmission coefficient of the amplitude of the mirror 7 . $\Phi_{\text {ref }}\left(l_{\text {ref }}\right)$ is the accumulated phase during propagation of the radiation along the optical path from the laser to the coherent detector, while $k_{r e f}$ is the optical transmission of the reference channel. It is evident that the total optical path 
of the reference signal $l_{\text {ref }}$ as counted from the output of the laser 2 in the basic station will be:

$$
l_{\text {ref }}=l_{\text {circ }}+2 l_{f i b},
$$

where $l_{\text {circ }}$ is the optical path in the circulator 3 from its input 3 a to its output $3 \mathrm{c}$ together with the lead-in/lead-out fibers from the laser 2 and to the detector 4 which in our case is evidently much shorter than the optical path of the fiber $l_{f i b}$, so $l_{f i b}>>>l_{\text {circ }}$, thus

$$
l_{\text {ref }} \approx 2 l_{f i b} .
$$

The useful signal carrying the information is described by the electric field $E_{\text {sig }}(t)$ and is formed by the wave from laser 2 and propagating along the fiber 5 , i.e., by the reference wave formed from the same laser wave and following the same path distance. After the semitransparent mirror 7 the field is written as:

$$
E_{\text {sig }}^{\prime}(t)=k_{\text {mir }} k_{\text {sig }}^{\prime} E_{0} \exp \left[-j\left(\omega_{\text {las }} t+\varphi_{\text {las }}(t)+\Psi_{\text {sig }}\left(l_{\text {sig }}^{\prime}\right)\right)\right],
$$

where $\Psi_{\text {sig }}\left(l_{\text {sig }}^{\prime}\right)$ is the phase of the signal accumulated along the optical path from the laser to the mirror $7, l_{s i g}^{\prime}=\left(l_{\text {circ }} / 2\right)+l_{f i b}$ and $k_{s i g}^{\prime}$ is the optical transmission coefficient along the same path in one direction. After the mirror 7 the wave traverses consecutively the fiber 8 of the acoustic sensor 6 of a total length $l_{\text {sens }}$ and after passing the polarization controller 9 is reflected by 10 whose reflection coefficient is $r_{m i r}$. The wave returned by the reflector 10 traverses again the sensor accumulating the same path $l_{\text {sens }}$ and through the semitransparent mirror 7 enters the optical fiber 5 across the same surface as the reference signal. After that the optical paths of both the sensor and the reference signal down to the coherent detector coincide. Correspondingly, their common optical path of the sensor signal $l_{s i g}$ which carries the information about the acoustic signal $U_{a c}(t)$ is:

$$
l_{\text {sig }}=l_{\text {circ }}+2 l_{f i b}+2 l_{\text {sens }} .
$$

From (5a) and (7) we obtain that the optical path difference between the signal and the reference wave is:

$$
\Delta l=l_{\text {sig }}-l_{\text {ref }}=2 l_{\text {sens }} .
$$

Equation (8) holds for arbitrary lengths of the lead-in/lead-out fiber 5, i.e. does not depend on how far from the basic source/interrogation unit 1 the fiber sensor module is located. In this way the proposed system minimizes the effects for possible unfavorable influences on the way from the source/interrogation unit to the sensor which is of greatest importance for high phase stability and 
detection efficiency particularly needed for the implementation of monitoring systems of remote sensors located at greater distances. From the above expressions we obtain an expression for the optical field on the photodetector in the signal channel:

$$
E_{\text {sig }}(t)=k_{\text {mir }}^{2} r_{m i r} . k_{\text {sig }} E_{0} \exp \left[-j\left(\omega_{\text {las }} t+\varphi_{\text {las }}(t)+\Phi_{\text {sig }}\left(l_{\text {sig }}\right)\right)\right],
$$

where $\Phi_{s i g}\left(l_{s i g}\right)$ is the total phase accumulated during the propagation of the wave from the laser 2 back to the photodetector $4, k_{s i g}=k_{r e f}+k_{\text {sens }}$ is the total optical transmission in the signal channel, $k_{\text {sens }}$ being the transmission in the acoustic sensor with $k_{\text {sens }} \ll k_{r e f}$ because of the short fiber length in sensor coil, and correspondingly $k_{\text {sig }} \sim k_{\text {ref }}$.

The introduction of information in the acoustic signal $U_{a c}(t)$ is based on the well know acousto-optic effect [8], which consists in the change of the refractive index in proportion with the imposed mechanical stress $\varepsilon_{a c}(t)=K_{e m} U_{a c}(t)$ caused by the acoustic wave, i.e.:

$$
n_{o p}[\varepsilon(t)]=n_{o p}\left[\left(U_{a c}(t)\right)\right] \sim n_{o p}^{0}+K_{e m} U_{a c}(t),
$$

where $n_{o p}^{0}$ is the average index of refraction, while $K_{e m}$ is the electromechanical coefficient. The changes of the index of refraction caused by the acoustic wave lead to changes of the speed of light in the sensing fiber section. The optical path $l_{\text {sens }}$ in the sensing fiber section thus becomes time dependent:

$$
l_{\text {sens }}(t)=l_{\text {sens }}^{0}+\Delta l_{\text {sens }}^{a c t v}\left[U_{a c}(t)\right],
$$

where $l_{\text {sens }}^{0}$ is the total optical path of the sensing fiber section, while $\Delta l_{\text {sens }}^{a c t v}\left[U_{a c}(t)\right]$ is the time dependent change in the part $l_{\text {actv }}<l_{\text {sens }}^{0}$ of the sensing fiber subjected to the acoustic field

$$
\Delta l_{\text {sens }}^{a c t v}\left(U_{a c}(t)\right)=l_{\text {actv }} K_{e m} U_{a c}(t) .
$$

The detected coherent signal $V_{\text {out }}(t)$ at the output 4.1 of the detector 4 is described by the well-known expression:

$$
V_{\text {out }}(t) \sim\left\langle E_{\text {sig }}(t) * \tilde{E}_{\text {ref }}(t)\right\rangle_{\tau_{\text {as }}},
$$

where $\tilde{E}_{r e f}(t)$ is the complex conjugated reference signal, while complex $\tau_{a}$ is the integration time constant of the acoustic signal. As a result, we obtain for the detected signal the expression:

$$
V_{\text {out }}(t) \sim k_{\text {mir }}^{2} r_{\text {mir }}\left(1-k_{\text {mir }}\right) k_{\text {sig }} k_{\text {ref }} E_{0}^{2} \exp (\Delta \Phi) .
$$


For the phases in equation (8) we obtain:

$$
\Delta \Phi=\Phi_{\text {sig }}\left(l_{\text {sig }}\right)-\Phi_{\text {ref }}\left(l_{\text {ref }}\right)=\frac{2 \pi}{\lambda_{\text {las }}} 2 l_{\text {sens }}
$$

i.e., the detected signal at the output 4.1 depends only on the optical path length of the sensing part of the acoustic sensor 6 irrespective of how remote it is from the basic unit. Using the expressions for $l_{\text {sens }}$ we obtain:

$$
\Delta \Phi=\varphi_{\text {sens }}^{0}+\frac{4 \pi}{\lambda_{\text {las }}} l_{\text {sens }}^{a c t v} K_{e m} U_{a c}(t)
$$

where $\varphi_{\text {sens }}^{0}=4 \pi l_{\text {sens }}^{0} / \lambda_{\text {las }}$. The expression for the useful output signal becomes:

$$
V_{\text {out }}(t) \sim K_{\text {eff }} k_{\text {sig }} k_{\text {ref }} E_{0}^{2} \mathrm{Z}_{\varphi} \exp \left[\frac{4 \pi}{\lambda_{\text {las }}} l_{\text {sens }} K_{\text {em }} U_{a c}(t)\right],
$$

with $K_{e f f}=k_{m i r}^{2} r_{m i r}\left(1-k_{m i r}\right)$.

Due to the low values of the coefficient $K_{e m}$ and the amplitude of the acoustic signal $U_{a c}(t)$ we limit the expansion into series of the exponent in expression (11) to its second term, and as a result we obtain an expression to evaluate the variable component of the output signal which is proportional to the amplitude of the acoustic signal:

$$
V_{\text {out }}(t) \sim V_{0} U_{\text {ac }}(t), \quad V_{0} \sim 4 \pi K_{\text {eff }} k_{\text {sig }} k_{\text {ref }} E_{0}^{2} \mathrm{Z}_{\varphi} \lambda_{\text {las }}^{-1} l_{\text {sens }} K_{\text {em }} .
$$

We can assume here that $\mathrm{Z}_{\varphi}=\exp \left(\varphi_{\text {sens }}^{0}\right)=$ const, based on the considerations that the elements of the acoustic sensor 6 (mirror 7 , sensing fiber, polarization controller and reflector 10 are encapsulated in a metallic box and are affected by ambient temperature changes, which compared to the time response of the detection system are extremely low in underwater deployment. By fine tuning the polarization controller 9 we can achieve that $\varphi_{\text {sens }}^{0}=2 \pi m$, $m=1,2,3 \ldots$ and hence $\mathrm{Z}_{\varphi}=1$. In this case for the internal loop 7-8-9-10-98-7 the resultant Jones matrix is unitary and reproduces the input polarization at mirror 7. Thus, the sensor and reference signals will be in phase at the output of the mirror 7. Due to the equality of the sensor and reference optical paths, the latter will arrive in phase at the photoreceiver so the detected signal will be at a maximum. The coefficients $k_{m i r}$ and $r_{m i r}$ can be optimized so that the coefficient $K_{\text {eff }}$ in expression (15) to be at maximum, in which case the output signal will equally be at a maximum for a given level of laser power. The electric signal $V_{a c}(t)$ containing the information for the acoustic signal $U_{a c}(t)$ is passed to the interrogation unit of the monitoring system for 
processing and decision making on the presence of an object, emitting the acoustic wave $U_{a c}(t)$.

The advantages of the scheme from Fig. 1 can be summarized as follows:

1) It ensures a perfect match between the optical paths of the sensor and reference signals outside the acoustic sensor, thus permitting the lead-in/leadout fiber to have lengths of 10-20 km and above and by reducing the requirements to the laser to those of coherence lengths of several meters which is the case for most of the commercially available lower cost lasers for Coarse Wavelength Division Multiplexing (CWDM). This in turn makes it possible to create highly efficient multiplexed coherent systems for acoustic control of remote wide marine and land areas;

2) Simplicity of the whole optical system including the remote acoustic sensor which does not need electrical power to function and the transmitter/receiver interrogation unit is located ashore in a closed cabinet. For lasers in the $\mathrm{C}$ (conventional) optical communication band (1530 $\mathrm{nm}$ to $1565 \mathrm{~nm}$ ) for which a single mode fiber has an attenuation of less than $0.15 \mathrm{~dB} / \mathrm{km}$, a sensor at $20 \mathrm{~km}$ from the basic station will accumulate $6 \mathrm{~dB}$ of power losses thus leaving sufficient margin for additional multiplexing.

Figure 2 presents a next development of a time multiplexed sensor system where it is of simultaneously functioning independent parallel acoustic sensors. The multiplexed system consists of a transceiver/receiver block 1 including a single frequency laser diode 1 with an external electro-optic modulator 2.0, an optical circulator 3 and a coherent optical detector 4 with time demultiplexing, as well as a lead-out/lead-in finer 5 , a $1 \times m$ splitter 12 with outputs from 12.1 to $12 . m$ to the corresponding secondary lead-in/lead-out fibers from 5.1 to $5 . m$ with different lengths and the corresponding outputs to a series of $m$ acoustic sensors 6.1 to $6 . m$ with input 7.1 to $7 . m$ and output 10.1 to $10 . m$ reflectors, sensor coils 8.1 to $8 . \mathrm{m}$, and polarization controllers 9.1 to $9 . \mathrm{m}$.

The system from Fig. 2 is analogous to that from Fig. 1. The reference signal from the laser 2.0 propagating along the common fiber 5 is divided by the splitter 12 and continues along the corresponding secondary lead-in/lead-out fibers 5.1 to $5 . m$ to the acoustic sensors from 8.1 to $8 . \mathrm{m}$. For an appropriate choice of the secondary fiber lengths, the sensor and reference pulses from each of sensors arrive independently to the coherent detector at fixed delays and are processed separately by the interrogation system.

The advantages of this scheme are as follows:

1) The possibility to cover a larger surveilled area which is as a whole at a large distance from the central station; 


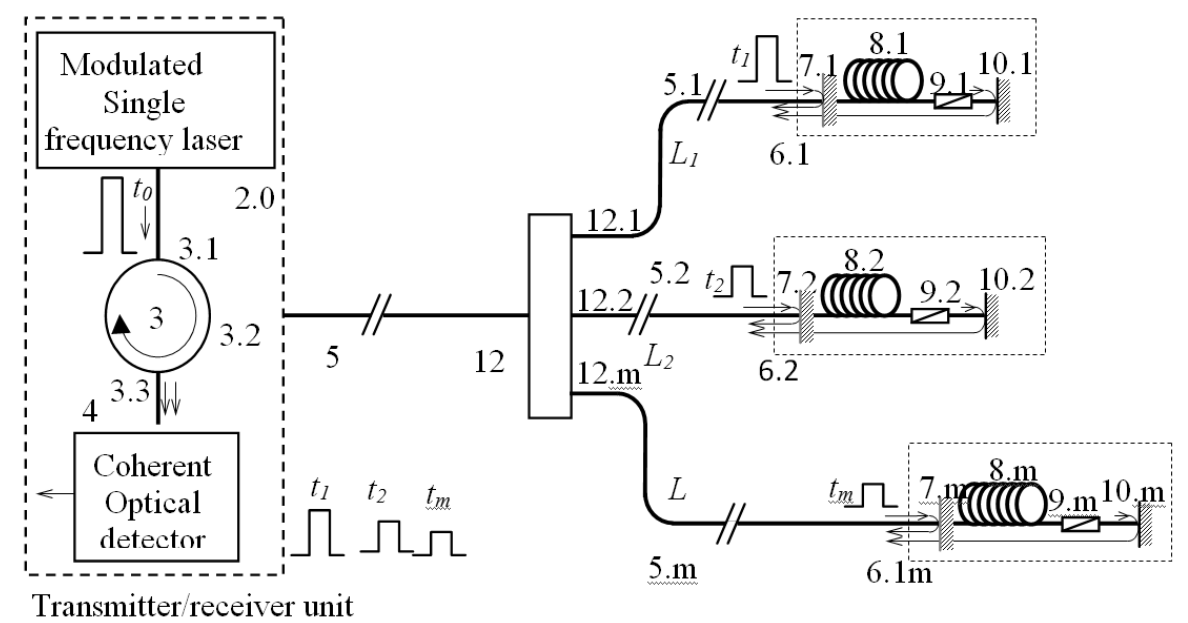

Fig. 2. Basic arrangement of a passive time division multiplexed sensor system with parallel acoustic sensors

2) The acoustic sensors after the splitter can be installed in an arbitrary arrangement depending on the structure of the system of monitoring;

3) A single lead-in/lead-out fiber is used since the sensors are inherently reflective;

4) A greater reliability if certain sensors fail;

5) Lower cost optical communication components are used.

\section{POSSIBLE APPLICATIONS OF THE DEVELOPPED FIBER OPTIC ACOUSTIC SYSTEMS FOR MARITIME AREA MONITORING}

The results from the analysis of the above presented arrangements of fiber optic monitoring systems of wide maritime areas [9] can find applications in the development of novel passive stationary systems for complex acoustic monitoring of large maritime and land areas (above $100 \mathrm{~km}^{2}$ ) that do not need electric power, avoid radio transmission and are low-cost.

The proposed arrangements allow for different geometry and with some modifications of different topologies depending on the particular geometry of the application.

The fact that electric power is needed only for the land-based basic unit, while the sensor system is all optical, means that once deployed on the bottom of the water basin the fiber network becomes virtually undetectable since only 
dielectric and plastic components are used and the metal parts of connectors and couplers are with millimeter dimensions. As the signals are optical, they emit no radio waves and are immune to radio jamming and electromagnetic noise. Suitably positioned sensors in a network can determine the location of the intruder by measuring time delays and using triangulation, pattern recognition techniques and can track several different moving objects by detecting their acoustic emissions and signatures. This allows maximum efficiency and versatility of monitoring.

The principle of operation of the above-described system was experimentally tested for different lengths of the lead-in/lead-out fiber 5 between the basic unit and the fiber sensor, namely $1 \mathrm{~km}, 3 \mathrm{~km}, 5 \mathrm{~km}$ and $10 \mathrm{~km}$. In all cases a stable functioning of the laser, the modulator and the sensor unit was observed and the acoustic signals were with sufficient signal-to-noise ratio which did not decrease with fiber length 5 . The sensing fiber was coiled on a $10 \mathrm{~cm}$ diameter drum which was excited using a piezo-electric transducer activated by a sine signal. We estimated experimentally the acoustic signal transmission quality at the passing throughout the entire fiber optical system of length exceeding $10 \mathrm{~km}$ between the more distant sensor 6 (see Fig. 1) and the position of the coherent optical detector 4 on the output 4.1 (Fig. 1). To this purpose we compared (see Fig. 3(a) the phase differences between the reference signal from a signal generator on the sensor 6 and the output signal on the output 4.1. As seen, both the signals (the input and the output) display very stable temporal phase stability. In addition, the frequency spectrum of the output signal is shown in the Fig. 3(b). It is evident the output signal from the 4.1 channel demonstrates a single frequency spectral line.



(a)

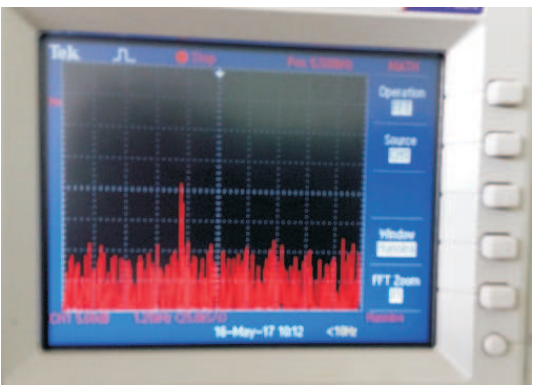

(b)

Fig. 3 
As a conclusion of this item, we can state the acoustic fiber optical system here described is capable to provide high quality acoustic signals to be applied in modern information system for detection of different underwater targets over large coastal sub-water areas, as well as for determination of their coordinates and the long-distance prevention of their activities within the monitored coastal areas by the system developed.

\section{CONCLUSION}

The high sensitivity of the developed acoustic fiber optic multi-sensor systems is achieved by using coherent detection with an accurate equalizing of the optical path between the sensor and reference signals to achieve high efficiency. These are necessary conditions to realize monitoring systems of areas located at great distances from the central station. It has been shown that the coherence length of the single frequency laser must be approximately equal to the fiber sensing section length $(\sim 10 \mathrm{~m})$ which is much less than the distance between the basic unit transmitter/receiver unit and the fiber acoustic sensor $(\sim 10 \mathrm{~km})$.

This allows by an appropriate choice of the single frequency laser and its coherence length to create unique time (TDM) and wavelength division multiplexed (WDM) fiber sensor networks of independent acoustic fiber sensors based on coherent detection. A single sensor and a network sensor arrangement were described that allow different applications in the monitoring of large marine and land areas. The network sensor system can monitor simultaneously a large number of surface and underwater moving vessels by detecting the emitted acoustic noises. The latter can be directly identified by an operator, applying an appropriate signal processing algorithm.

\section{REFERENCES}

[1] Malcolm Paul Varnham, Erhard Lothar Edgar Kluth, Phillip Sam Bull, and John Luscombe, Seismic Sensor With Interferometric Sensing Apparatus, US Patent No.: US 6,195,162 B1 Feb. 27, 2001.

[2] Henry F. Taylor, Ta-Wei Kao, James Gardner, William N. Gibler, Robert A. Atkins, Chung E. Lee, Vlctor P. SwWenson, Matthew Spears, and Robert X. Perez, Fiber Optic Fiber Fabry-Perot Interferometer Diaphragm Sensor and Method of Measurement, US Patent No.: US 6,281,976 B1 Aug. 28, 2001.

[3] Charles M. Davis, Fiber Optic Interferometric Thermometer With Serially Positioned Fiber Fiber Optic Probes US Patent No.: 4,755,668, Jul. 5, 1988. 
[4] Charles M- Davis, Fiber Optic Interferometric Thermometer, Patent Number: 4,868,381 Sep. 19, 1989.

[5] Alan D. Kersey And Anthony Dandridge, Serial Interferometric FiberOptic Sensor Array, US Patent No.: 4,889,986, Dec. 26, 1989.

[6] Erlend Ronnekleiv, Elimination of Polarization Fading In Unbalanced Optical Measuring Interferometers, Patent No.: US 6,856,401 B1, Feb. 15, 2005.

[7] Byoung Y. Kim, Moshe Tur, Janet L. Brooks, Kenneth A. Fesler, And Herbert J. Shaw, Distributed Sensor Array And Method Using A Pulsed Signal Source, Patent Number: 4,770,535, Sep. 13, 1988.

[8] H. Alben and C. Guillemet, Photoelsticity of Glass, Ch. 3 Basic Photoelasticity, Springer-Verlag, Berlin (1993).

[9] D. V. Stoyanov, A. S. Popov, T. A. Eftimov, and S. Vodenicharov, Acoustic Fiber Optics Sensing System, Patent BG: G 01 B 9/02 (2006.01), 2020.

Received March 10, 2021

Engineering Sciences, LVIII, 2021, No. 1 